\section{Research Square}

Preprints are preliminary reports that have not undergone peer review.

They should not be considered conclusive, used to inform clinical practice, or referenced by the media as validated information.

\title{
Application of chloroplast genome to resolve the taxonomy and phylogenetic relationships of invasive dioecious weeds in Amaranthus (Amaranthaceae)
}

Han Xu ( $\nabla$ xuhangin@gmail.com)

\section{Research article}

Keywords: Phylogenetic relationship, Chloroplast genome, Invasive alien plants, Amaranthus palmeri S. Watson, Amaranthus arenicola I. M. Johnston, Amaranthus tuberculatus (Moq.) J.D. Sauer, Amaranthus subgen. Acnida (L.) Aellen ex K. R. Robertson

Posted Date: December 19th, 2019

DOI: https://doi.org/10.21203/rs.2.19304/v1

License: (c) (i) This work is licensed under a Creative Commons Attribution 4.0 International License. Read Full License 


\section{Abstract}

Backgroud: Amaranthus palmeri, A. tuberculatus and A. arenicola are alien invasive dioecious amaranths originated from North America which have similar morphology and complex taxonomic relationship with their relatives. To search for effective molecular methods and accurate species boundary for detecting the alien invasive species, we sequenced whole chloroplast genome of 6 amaranths species, of which A. palmeri , A. arenicola , A. retroflexus and A. dubius are the first reports.

Results: The complete chloroplast genome of 6 species has a circular molecular structure of 150,454 to 150,939 bp in length with $36.6 \%$ of GC content and contains a total of 134 genes, including 89 protein-coding genes, 37 tRNA genes, and 8 rRNA genes. There are a total of 802 parsimony-informative (PI) sites within genes and intergenic spacers. The rpl22-rps19, ndhG-I , rpl 32- trnLUAG , trnPUGG-psaJ and ccsA - ndhD are the hotspots in the genus. And the 1,601 bp fragment from rpl32 to psaC has contained maximum variants with $82 \mathrm{PI}$ sites. A. arenicola differs from A. tuberculatus with $19 \mathrm{PI}$ sites located in 14 genes and spacers separately. The regions for differentiate A. dubius, A. hypochondriacus and A. caudatus of the Hybrid complex only fasten on 2 coding genes and 5 intergenic spacers. The patristic distances (0.00001-0.00005) among the three species are approximate to the distance (0.00005) between individuals of $A$. tuberculatus . Conformed to dioecious and monoecious distinctions but different with previous phylogenetic studies, A. palmeri clustered with A. arenicola and A. tuberculatus and formed a stable clade of subgen. Acnida .

Conclusion: The chloroplast genome has played a role in offering enough information for discrimination and phylogenetic relationship among the Amaranthus subgen. Acnida. The most valuable regions of chloroplast genome in Amaranthus are intergenic spacers and could differentiate A. arenicola from A. tuberculatus better. Subsequently, much more Amaranthus species should be sequenced and analyzed complementally in the future.

\section{Background}

The genus Amaranthus comprises at least 60 species, most of them are annual weeds and distributed throughout the world's temperate and tropical regions (Mosyakin \& Robertson, 2003). Several amaranths are economic crops, such as A. hypochondriacus L. and A. caudatus L. (Sauer, 1950), and a well-known vegetable and horticultural plant A. tricolor L. (Advisory Committee on Technology Innovation, 1984). In contrast to this, A. palmeri S. Watson, A. tuberculatus (Moq.) J.D. Sauer and A. retroflexus L. are famous invasive weeds (Ward et al., 2013; Costea, 2005; Steckel \& Sprague, 2004). These weeds not only invade new habitats easily and cause environmental and agricultural damages, but are trouble subgroups hard to identify, especially their small similar, about $1 \mathrm{~mm}$ diameter seeds (Ward et al., 2013; Costea, 2005; Steckel \& Sprague, 2004; Xu et al., 2014). These invasive weed seeds often been carried by grain trades through different countries and continents, and have been intercepted from imported grains and monitored on the ports in China (Xu et al., 2012 , 2013). They have brought huge environmental and agricultural damage risks. But their identification always is an intractable problem.

In addition, the genus Amaranthus is divided into 3 subgenera presently: Amaranthus subgen. Acnida (L.) Aellen ex K. R. Robertson, Amaranthus subgen. Amaranthus and Amaranthus subgen. Albersia (Kunth) Gren. \& Godr., according to dieocious or monoecious and the tepal or stamen number (Mosyakin \& Robertson, 1996). A. palmeri, A. tuberculatus, and A. arenicola are dioecious, belong to Amaranthus subgen. Acnida (L.) Aellen ex K. R. Robertson, and often be misidentified for their similar morphology (Sauer, 1955, 1972). These three dioecious amaranths all originated from North America and have spread widely in agricultural fields and other disturbed areas (Sauer, 1955, 1972).

Palmer amaranth is one of a distinct subgroup of dioecious species within Amaranthus which hybridization among different species has been widely reported (Trucco et al., 2007; Steckel, 2007). Gaines et al. (2012) found the highest levels of successful hybridization (up to 0.4\%) occurred between Palmer amaranth and spiny amaranth, with this cross producing viable and fertile F1 progeny (Gaines et al., 2012). All like that are often of frequent occurrence among different amaranths. Especially the confused relationships among the waterhemp complex (A. tuberculatus var. rudis (J.D. Sauer) Costea \& Tardif and A. tuberculatus (Moq.) J.D. Sauer var. tuberculatus) with A. arenicola, that prompts us to get more details about these taxa through a new perspective.

The chloroplast genome has been studied in Amaranthus, but mainly referring to the species under Amaranthus subgen. Amaranthus and subgen. Albersia, and get better effectiveness on the phylogeny than common DNA barcodes ITS, matK, and rbcL, but poorly results were gotten at resolving the Hybrid complex (A. hyrbidus, A. caudatus, A. hypochondriacus, A. quitensis, A. dubius, A. cruentus) except the GBS methods (Chaney et al., 2016; Wu \& Blair, 2017; Viljoen et al., 2018). ITS sequence is enough for most species of Amaranthus, but limited on the dioecious specie and the Hybrid complex (Xu et al., 2017a,b).

In previous studies, a high ITS sequence homology degree between Palmer amaranth and spiny amaranth has been found, and A. palmeri was separated from subgen. Acnida and then merged into subgen. Amaranthus (Xu et al., 2017a; Kirkpatrick, 1995). This conclusion is opposite to traditional taxonomy opinion. Whether the taxonomic status of subgen. Acnida is tenable needs our further studies. In our papers, we focus on seeking appropriate identified regions for amaranths based on the chloroplast. In addition, we try to find out a new viewpoint for resolve our previous doubts about the status of subgen. Acnida.

\section{Results}

\section{Genomic features}

The complete chloroplast genome of six Amaranthus spp. has a circular molecular structure of 150,454 to 150,939 bp in length with $36.6 \%$ of GC content. It has a large single copy (LSC) region of 83,747 to 84,340 bp and a small single copy (SSC) region of 17,898 to 18,044 bp, separated by a pair of identical inverted repeat regions (IRs) of 24,519 to 24,582 bp each (Table S1). The chloroplast genome contains a total of 134 genes, including 89 protein-coding genes, 37 tRNA genes, and 8 rRNA genes, 19 of which were duplicated in the inverted repeat regions (Table S2). The rps 12 not only lie in the LSC, but 
duplicated in the IRs. 14 genes (rps12, rps16, atpF, rpoC1, ndhB, petB, petD, ndhA, trnK-UUU, trnI-GAU, trnA-UGC, trnG-UCC, trnL-UAA and trnV-UAC) contained a single intron, while ycf3 and clpP harbored two introns separately (Table S3).

\section{Comparative genomic analysis and hotspot regions for identification}

After aligned, sequence variablitiy was due solely to the presence of single nucleotide polymorphism (SNP) and indels. No gene rearrangements of genome or differences in gene content were observed. There are a total of $802 \mathrm{PI}$ sites among 134 genes and 132 intergenic spacers (Table 1 and S3). Analysis of the distribution of genetic variability within the chloroplast genome of Amaranthus revealed that the most variable region is the Small Single Copy Unit (SSC) with 1.12 percent of nucleotide variation (Table 1). And the variation frequency of intergenic spacers is 1.05 percent much more than 0.32 percent of the gene regions (Table 1).

Among the 134 genes, the highest frequency of polymorphism was found in rp/16 (1,385 bp, 2.671\%) and psbT (102 bp, 1.961\%) (Table S3). On the other side, rp/22-rps19 (132bp, 9.091\%), ndhG-I (146 bp, 6.849\%), rp/32-trnLUAG (1,177 bp, 5.523\%), trnPUGG-psaJ (422 bp, $5.213 \%)$ and ccsA-ndhD (201 bp, $5.473 \%$ ) are the biggest concentration of polymorphic intergenic spacers (Table S4). And the 1,601 bp fragment from rp/32 to psaC has contained maximum variants with 82 SNPs (Table S4).

The hotspots for discriminate $A$. arenicola with $A$. tuberculatus mainly concentrate in 6 genic regions (ndhA, rp/16, ccsA, matK, atpF and ycf1) and 8 intergenic spacers (psbA-trnKUUU, trnDGUC - trnYGUA, ndhC - trnVUAC, trnEUUC - trnTGGU, trnTUGU - trnLUAA, atpB - rbcL, rbcL - accD, trnWCCA - trnPUGG, trnPUGG - psaJ and rps 15 - ycf1) each contains one or two PI sites (Table S3 and S4). The SNPs for differentiate the complex of $A$. dubius, $\underline{A}$. hypochondriacus and $A$. caudatus fasten on 2 coding genes (matK and $r p / 16$ ) and 5 non-coding spacers (trnHGUG - psbA, rpoB - trnCGCA, psbC - trnSUGA, trnGGCC-trnfMCAU and ndhF - rp/32) (Table S3 and S4).

\section{Phylogenetic tree}

The two phylograms constructed by whole chloroplast genome and $802 \mathrm{PI}$ sites are consistence basically (Figure 1 and S1). Conformed to traditional taxonomy opinions, $A$. palmeri together with $A$. arenicola and $A$. tuberculatus formed the clade of subgen. Acnida. A. arenicola has apart with $A$. tuberculatus by 19 SNPs. A. tricolor represents subgen. Albersia, has closer relationship with subgen. Acnida rather than subgen. Amaranthus. A. dubius is closer with A. hypochondriacus and A. caudatus than A. hybridus ssp. cruentus.

\section{Genetic distance}

According to patristic distances calculated by whole plastid genome and pairwise distance based on PI sites, 2 individuals of $A$. tuberculatus have small difference (0.00005) (Table S5). A. arenicola differs from $A$. tuberculatus with 19 SNPs and 0.00029 patristic distance value (Table S5). The complex of $A$. dubius, $A$. hypochondriacus and $A$. caudatus have poor differences $(0.00001-0.00005)$ and approximate to the distance (0.00005) between individuals of $A$. tuberculatus. (Table S5). And A. hybridus ssp. cruentus keeps away from the complex with above 0.0016 values (Table S5).

\section{Discussions}

\section{Discrimination of Amaranthus spp. based on the chloroplast genome}

Concluded from the results, the phylogram based on the chloroplast genome showed more information about relationships and species boundary among Amaranthus than the ITS, rbcL and matK. Some species with clear demarcation could identified by ITS, but the similar relatives or the complex cannot get a good results for their high consistence on ITS (Xu et al., 2017a). Different with previous studies, there are five intergenic regions rp/22-rps19 (132bp, 9.091\%), ndhG-I (146 bp, 6.849\%), rp/32-trnLUAG (1,177 bp, 5.523\%), trnPUGG-psaJ (422 bp, 5.213\%) and ccsA-ndhD (201 bp, $5.473 \%)$ have offerred more informative phylogeny for Amaranthus, and much better than $n d h D, r p o C 2$, atpE and $r p / 22$ (only $1 \%$ to $1.9 \%$ ) found by Viljoen et al. (2018). That's for the sequences they used to align and analyze came from the species among Amaranthus subgen Amaranthus and Amaranthus subgen. Albersia, and not involved in three subgenera, and the hotspots will be limited. Even expanded research taxa, the valuable informative sites still scatter in different genic and intergenic regions. As for the confused taxa, if we want to set apart A. arenicola from A. tuberculatus, or distinguish the species of the Hybrid complex, that needs at least 6 genic and 13 intergenic regions. Efficient molecular markers based on these SNPs should be developed for rapid identification. And more different geographical individuals of one species ought to be used in verify the availability of these variable sites.

\section{Taxonomy of Amaranthus subgen. Acndia}

From the previous classification system of Amaranthus to date, many scholars have put forward different revision suggestions. The primary taxonomic system for Amaranthus was revised by Mosyakin \& Robertson (1996) and for Amaranthus subgen. Acnida was classified by Sauer (1955). But under subgenus level, the divisions of sects or species boundary are not clear and controversial and often rectified by other scholars.

In Amaranthus subgen. Acnida, A. tuberculatus is a complex was formed of two varieties: A. tuberculatus var. rudis (J.D. Sauer) Costea \& Tardif and A. tuberculatus (Moq.) J.D. Sauer var. tuberculatus, and has more widely diversity genetic structures and viable morphology (Mosyakin \& Robertson, 2003; Pratt \& Clark, 2001). The species can hybridize in the wild with other relatives of subgen. Acnida, even with monoecious species vest in Amaranthus subgen. Amaranthus (Costea et al., 2005; Waselkov \& Olsen, 2014). That's why the phylogenetic status or species boundary are always unclear, in spite of they have several distinct characteristics for discern on morphology. Chances are the staminiferous plants could be shared widely among the different dioecious species. Especially, they all originate from the same regions and distribute overlapped. That's might be $A$. arenicola is very close with $A$. tuberulatus, and both of them are hard to distinguish. 
A. palmeri is very close with A. spinosus which belonging to Amaranthus subgen. Amaranthus nothosect. Dubia Mosyakin \& K. R. Robertson (Mosyakin \& Robertson, 1996; Xu et al., 2017a,b; Kirkpatrick, 1995). In prior studies, A. palmeri was divided from subgen. Acnida and attributed to subgen. Amaranthus based on the ITS and ALS (Xu et al., 2017a,b; Kirkpatrick, 1995; Xu, 2019, Unpublished data). The taxonomic status of subgen. Anicda seem to be broken up. But in our studies, $A$. palmeri has a longer genetic distance with subgen. Amaranthus, and much more close with $A$. arenicola and $A$. tuebrculatus. The phylogenetic status of subgen. Acnida seem to be stable and form an independent clade on the plastid genome.

\section{Phylogenetic status of Amaranthus subgen. Amaranthus}

Another group is the Hybrid complex which is the most studied taxa (Sauer, 1967). Erika Viljoen et al. (2018) and Lindsay Chaney et al. (2016) all came to a poor conclusion gotten by chloroplast data for resolving recent evolution of the complex (Chaney et al., 2016; Viljoen et al., 2018). Only the SNPs acquired from whole genome (GBS) get a better result for their taxonomy (Wu \& Blair, 2017). About the Hybrid complex, our research continues to be unsatisfied, and just found 2 genes and 5 intergenic spacers could be served as markers for the next studies. Besides, the genetic distance (0.00001-0.00005) among the three species are approximate to the distance $(0.00005)$ between individuals of $A$. tuberculatus. It means that the three species might be segmented into subspecies or varieties under $A$. hybridus instead of an independent species more reasonably.

\section{Conclusions}

All above conclusions have accounted for the genus is a trouble taxa. The chloroplast genome has played a role in offering enough information for discrimination and phylogenetic relationship among the Amaranthus, especially the Amaranthus subgen. Acnida. That's the first reports for the subgen. Acnida based on the plastid. Subsequently, much more Amaranthus species should be sequenced and analyzed complementedly in the future.

\section{Methods}

\section{Plant samples, DNA extraction, and sequencing}

Fresh leaves of each individual of $A$. palmeri, $A$. arenicola, $A$. retroflexus, $A$. dubius and two accessions of $A$. tuberculatus var. tuberculatus were sampled from imported borders in China and dried using silica gel. Voucher specimens (Table S1) were deposited at the plant quarantine institute of Chinese Academy of Inspection and Quarantine (CAIQ) (Beijing, China). All the samples used in the experiment are legal evidence samples from each port submitted for species identification. The identification is part of CAIQ's laboratory functions. The basis for identification was the classification monographs of Amaranthus by Sauer (1972) and Mosyakin and Robertson (2003). Total genomic DNA was extracted from the silica-dried leaf tissues using Plant Genomic DNA Kit (Tiangen Biotech Co., China). Genomic DNA of each individual was indexed by a barcode and then pooled together with other samples for sequencing in one lane of HiSeq 2500 (Illumina) (Novogene, Beijing, China). The whole chloroplast genomes of $A$. hypochondriacus (GenBank accession No. MG836505), A. caudatus (NC040143), A. hybridus ssp. cruentus (MG836507) and A. tricolor (KX094399) were downloaded from NCBI (https://www.ncbi.nlm.nih.gov/).

\section{Genome assembly and annotation}

The paired-end sequencing data $(2 \times 150 \mathrm{bp})$ were used to assemble its complete chloroplast genome. Sequencing adapters and barcodes were trimmed and low quality reads with Q value $\leq 30$ removed. Trimmed paired end reads were mapped to the chloroplast sequence of $A$. hypochondriacus (MG836505), with default parameters. The reads were assembled using the Geneious Prime v. 2019.1.3 (Biomatters, Auckland, New Zealand). The consensus chloroplast sequence of four Amaranthus spp. was retrieved separately and used as a reference for a second round of mapping of itself reads in order to validate its consensus chloroplast sequence. All trimmed and quality-filtered sequence reads have been deposited in Genbank of NCBI. Non-mapped reads, which are assumed to be of non-plastid origin, were excluded from further analysis. The complete chloroplast genome sequence was annotated using the Geneious Prime v. 2019.1.3 (Biomatters, Auckland, New Zealand) by comparing with the genome of A. hypochondriacus (GenBank accession No: MG836505). The assembled and annotated Amaranthus spp. chloroplast genome sequence was deposited at NCBI (Table S1).

\section{Genome comparative analysis}

A comparative plot of full alignment with annotations of the 10 genomes of Amaranthus was produced by Geneious prime v.2019.1.3 software (Biomatters, Auckland, New Zealand), using the annotation of A. hypochondriacus (MG836505) as a reference. To analyze nucleotide diversity and parsimonyinformative (PI) sites, we extracted all the regions (including coding regions, introns, and intergenic spacers) after alignment, and put into Molecular Evolutionary Genetics Analysis (MEGA) v6.06 software (Tamura et al., 2011) for next phylogenetic analyses, patristic distance calculation and discern the hotspot regions. The extraction followed two criteria: (i) each gene and intergenic spacer; and (ii) mutation site with variant frequency above $20 \%$. The parsimony-informative $(\mathrm{PI})$ sites are much credible than single SNP for the discrimination of difficult groups and phylogenetic analyses.

\section{Whole-plastid genome tree and parsimony-informative $(\mathrm{PI})$ sites-based tree}

Chloroplast genomic phylogenetic analyses were performed based on 12 sequences of 11 species in Amaranthaceae. These sequences were aligned using the Geneious Prime v.2019.1.3 software (Biomatters, Auckland, New Zealand) and Neighber-Joining (NJ) analysis was conducted with Celosia trigyna L. (MN057637) and Alternanthera philoxeroides (Mart.) Griseb. (MK795965) as outgroups using Geneious Tree Builder and confidence for nodes determined using bootstrap analysis with 1000 replicates. In addition, we extracted all PI sites except indels of each gene and intergenic spacer from the whole chloroplast genome and formed a fasta forma file putting into MEGA v6.06 software (Tamura et al., 2011) for phylogenetic analyses and construct a phylogram based on the PI sites. 


\section{Declarations}

\section{Ethics approval and consent to participate}

Not applicable.

\section{Consent for publication}

Not applicable.

\section{Availability of data and materials}

The datasets generated and/or analysed during the current study are available in the GenBank repository, https://www.ncbi.nlm.nih.gov/.

\section{Competing interests}

The authors declare that they have no conflict of interest.

\section{Funding}

This work was supported by National agricultural standardization Project NBFW-14-2018, and the Basic Scientific Research Program of CAIQ (2014JK010区 2017JK038). The work involved in this study is part of the above projects on pest identification techniques.

\section{Authors' contributions}

$\mathrm{XH}$ designed the experiments and write the paper.

\section{Acknowledgements}

Thanks to Xiaoxia Zhang and Liansheng Xu of Institute of Botany, Chinese academy of sciences, for their technical guidance in the annotation of genome analysis. Thanks to the port staff who provided samples to the laboratory.

\section{References}

1. Advisory Committee on Technology Innovation. Amaranth, modern prospect for ancient crop. Report of an Ad Hoc Panel of the Advisory Committee on Technology Innovation Board on Science and Technology for International Development Office of International Affairs, National Research Council. Washington DC: National Academic Press; 1984.

2. Chaney L, Mangelson R, Ramaraj T, Jellen EN, Maughan PJ. The Complete Chloroplast Genome Sequences for Four Amaranthus Species (Amaranthaceae). Appl Plant Sci. 2016; 4(9): 1600063.

3. Costea M, Weaver SE, Tardif F. The biology of invasive alien plants in Canada. 3. Amaranthus tuberculatus (Moq.) Sauer var. rudis (Sauer) Costea \& Tardif. Can J Plant Sci. 2005; 85: 507-522.

4. Gaines TZ, Ward SM, Bekun B, Preston C, Leach JE, Westra P. Interspecific hybridization transfers a previously unknown glyphosate resistance mechanism in Amaranthus species. Evol Appl. 2012; 5: 29-38.

5. Kirkpatrick BA. Interspecific relationships within the genus Amaranthus (Amaranthaceae. Ph.D. dissertation. College Station). TX: Texas A \& M University; 1995. p. 87.

6. Mosyakin SL, Robertson KR. New infrageneric taxa and combinations in Amaranthus (Amaranthaceae), Ann Bot Fennici. $1996 ; 33: 275-281$.

7. Mosyakin S, Robertson KR. Amaranthus. Magnoliophyta: Caryophyllidae, part 1. In: Flora of North America Editorial Committee editors. Flora of North America North of Mexico, Vol. 4. New York: Oxford University Press; 2003

8. Pratt DB, Clark LG. Amaranthus rudis and A. tuberculatus, one species or two? J Torrey Bot Soc. 2001; 128: $282-296$.

9. Sauer JD. The grain amaranths: a survey of their history and classification. Ann Mo Bot Gard. 1950; 37: 561-632.

10. Sauer JD. Revision of the dioecious amaranths. Madroño. 1955; 13: 5-46.

11. Sauer JD. The grain amaranths and their relatives: a revised taxonomic and geographic survey. Ann Mo Bot Gard. 1967; 54: 103-137.

12. Sauer JD. The dioecious amaranths: a new species name and major range extensions. Madroño. 1972; 21: 426-434.

13. Steckel LE, Sprague CL. Common waterhemp (Amaranthus rudis) interference in corn. Weed Sci. 2004; 52(3): 359-364.

14. Steckel LE. The dioecious Amaranthus spp.: here to stay. Weed Tech. 2007; 21: 567-570.

15. Tamura K, Peterson D, Peterson N, Stecher G, Nei M, Kumar S. MEGA5: molecular evolutionary genetics analysis using maximum likelihood, evolutionary distance, and maximum parsimony methods. Mol Biol Evol. 2011; 28(10): 2731-2739.

16. Trucco F, Jeschke MR, Rayburn AL, Tranel PJ. Promiscuity in weedy amaranths: high frequency of female tall waterhemp (Amaranthus tuberculatus) $\mathrm{x}$ smooth pigweed (A. hybridus) hybridization under field conditions. Weed Sci. 2005; 53: 46-54.

17. Trucco F, Zheng D, Woodyard AJ, Walter AR, Tatum TC, Rayburn AL, Tranel PJ. Nonhybrid progeny from crosses of dioecious Amaranths: implications for gene-flow research. Weed Sci. 2007; 55: 119-122. 
18. Viljoen E, Odeny DA, Coetzee MPA, Berger DK, D.J.G. Rees DJG. Application of Chloroplast Phylogenomics to Resolve Species Relationships Within the Plant Genus Amaranthus. J Mol Evol. 2018; doi:10.1007/s00239-018-9837-9.

19. Ward SM, Webster TM, Steckel LE. Palmer Amaranth (Amaranthus palmeri): A Review. Weed Tech. 2013; $27: 12-27$.

20. Waselkov KE, Olsen KM. Population genetics and origin of the native north American agricultural weed waterhemp (Amaranthus tuberculatus, Amaranthaceae). AJB. 2014; 101 (10): 1726-1736.

21. Wu X-B, Blair MW. Diversity in Grain Amaranths and Relatives Distinguished by Genotyping by Sequencing (GBS). Front Plant Sci. $2017 ; 8: 1960$.

22. Xu H, Chen K, Ouyang Z-Y, Pan X-B, Zhu S-F. Threats of invasive species for china caused by expanding international trade. ES \& T. 2012; 46: 7063-4.

23. Xu H, Song Y, Fan X-H, Yang S-J, Wu Y. Invasion risk and suitability analysis of three kinds of Acnida weeds (Subgen. Acnida L.) in China. Plant Quarantine. 2013; 27(4): 20-23.

24. Xu H, Li Z-Y, Liao F, Liu Y. Seed morphological studies on the genus of Amaranthus in China. Plant Quarantine. 2014 ; 28 (2): $33-38$.

25. Xu H, Li Z-Y, Li J-S. Phylogenetic analysis of alien species of Amaranthus in China based on ITS sequences. Guihaia. $2017 \mathrm{a} ; 37$ (2): $139-144$.

26. Xu H, Zhao C-Y, Liu Y-B, Chen P-C, Li J-S. Taxonomic circumscription of Amaranthus palmeri, A. rudis and A. tuberculatus (Amaranthaceae) based on ITS sequences and SNP analysis. Plant protection. 2017b; 43(5): 128-133.

27. Xu H. Detecting alien invasive Amaranthus weeds using ITS and ALS and R ALS allele biotypes were founding from China Ports. Unpublished; 2019.

\section{Table}

Table 1 Variations within genes and intergenic regions among 10 whole chloroplast genomes of 9 Amaranthus spp. .

\begin{tabular}{|c|c|c|c|c|c|c|c|c|c|c|c|c|c|c|c|}
\hline \multirow[t]{2}{*}{ Reigon } & \multicolumn{6}{|c|}{ Coding regions } & \multicolumn{5}{|c|}{ Non-coding regions } & \multicolumn{4}{|c|}{ Whole chloroplast genome } \\
\hline & Length & SNP & Indel & Total & $\begin{array}{l}\text { Variable } \\
\text { frequency }\end{array}$ & Length & SNP & Indel & Total & $\begin{array}{l}\text { Variable } \\
\text { frequency }\end{array}$ & Length & SNP & Indel & Total & $\begin{array}{l}\text { Variable } \\
\text { frequency }\end{array}$ \\
\hline LSC & 56,218 & 176 & 60 & 236 & $0.42 \%$ & 30,110 & 205 & 105 & 310 & $1.03 \%$ & 86,328 & 381 & 165 & 546 & $0.63 \%$ \\
\hline IRa & 19,673 & 12 & 0 & 12 & $0.06 \%$ & 4,910 & 6 & 7 & 13 & $0.26 \%$ & 24,583 & 18 & 7 & 25 & $0.10 \%$ \\
\hline SSC & 15,509 & 88 & 4 & 92 & $0.59 \%$ & 2,990 & 51 & 64 & 115 & $3.85 \%$ & 18,499 & 139 & 68 & 207 & $1.12 \%$ \\
\hline $\mathrm{IRb}$ & 19,673 & 11 & 1 & 12 & $0.06 \%$ & 4,910 & 6 & 6 & 12 & $0.24 \%$ & 24,583 & 17 & 7 & 24 & $0.10 \%$ \\
\hline Total & 111,073 & 287 & 65 & 352 & $0.32 \%$ & 42,920 & 268 & 182 & 450 & $1.05 \%$ & 153,993 & 555 & 247 & 802 & $0.52 \%$ \\
\hline
\end{tabular}

*Note: The region length of genes and intergenic spacers includes gaps and originated from the consensus sequence aligned by 10 whole chloroplast genomes of 9 Amaranthus spp..

\section{Figures}




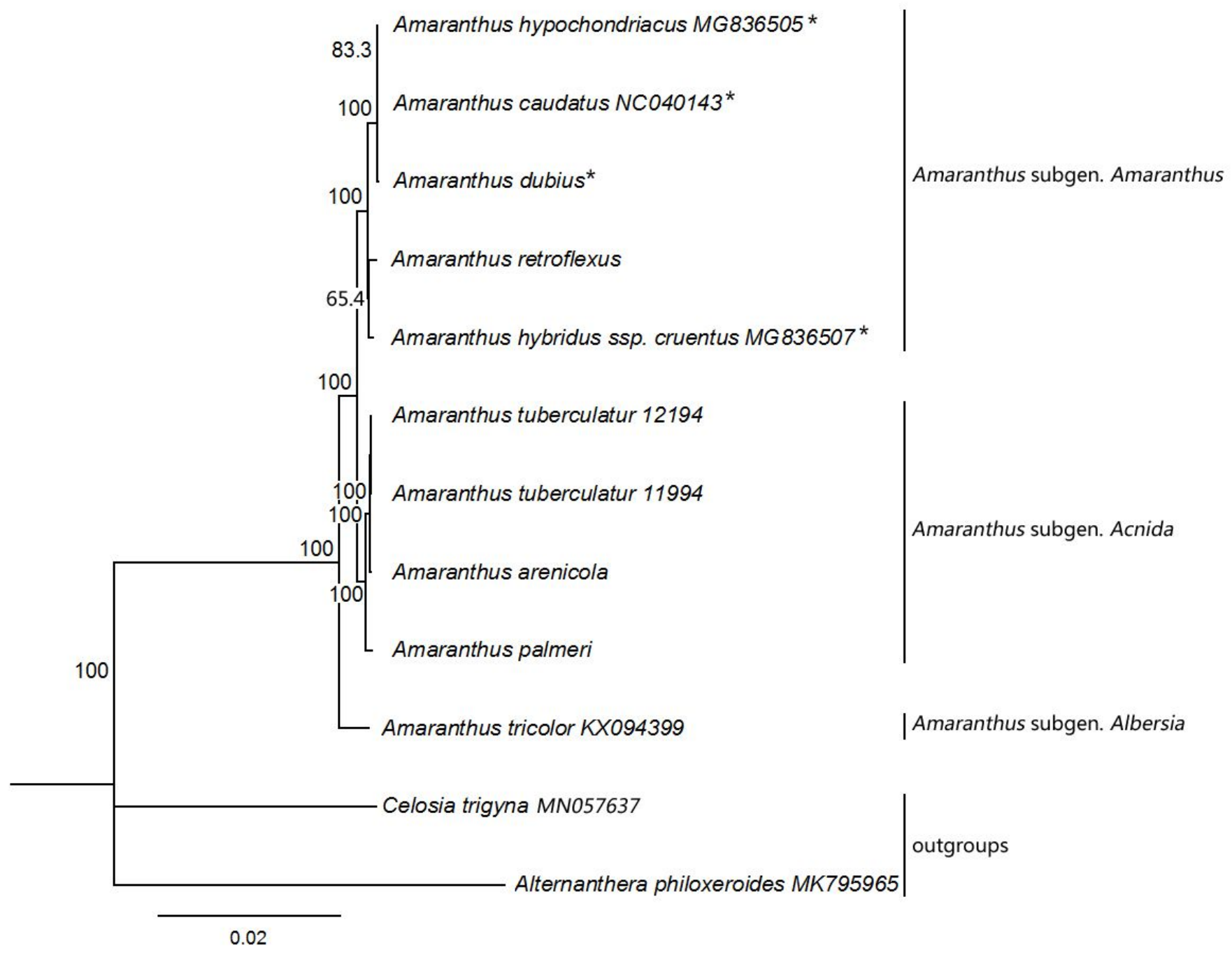

Figure 1

The Neighber-Joining ( $\mathrm{NJ}$ ) tree based on the 12 representative chloroplast genomes of family Amaranthaceae. The bootstrap value based on 1000 replicates is shown on each node. *The species of the Hybrid complex.

\section{Supplementary Files}

This is a list of supplementary files associated with this preprint. Click to download.

- Figures1.JPG

- TablesupplementaryS15.xlsx 